TRANSACTIONS OF THE

AMERICAN MATHEMATICAL SOCIETY

Volume 354, Number 2, Pages 837-852

S 0002-9947(01)02866-5

Article electronically published on July 12, 2001

\title{
ON INVERSION OF THE BESSEL AND GELFAND TRANSFORMS
}

\author{
MASAAKI FURUSAWA AND JOSEPH A. SHALIKA
}

\begin{abstract}
We construct the Plancherel measure corresponding to the Bessel model on the split special orthogonal group of odd degree $\geq 5$ and the Whittaker model on a connected split reductive group in general. As an application we prove the inversion formula which expresses the related integral transform in terms of the Satake transform.
\end{abstract}

\section{INTRODUCTION}

In this paper we will study spaces of functions on the groups of $F$-rational points of connected reductive algebraic groups defined over a non-archimedean local field $F$, which are related to certain unique models. Namely we study the space of functions related to the Bessel model on the split special orthogonal group $\mathrm{SO}(2 n+1)$ $(n \geq 2)$ and then the space related to the Whittaker model on a split connected reductive group in general.

We define the Fourier transform of functions in these spaces and then construct a measure on the unramified dual so that we have a Fourier inversion formula. Our construction of the corresponding Plancherel measure is modeled after Macdonald [5, which in turn was inspired by Harish-Chandra's work on the archimedean case, and it is based on the explicit formula for the Bessel model in [1] and the one for the Whittaker model in [3]. As an application of the Fourier inversion formula, we obtain the inversion formula for the corresponding Bessel and Gelfand transforms (cf. 2.3 and 3.3 below) in terms of the well-known Satake transform in the spirit of Yangbo Ye's papers [7, 8].

Although the results in this article seem to be of independent interest from the viewpoint of harmonic analysis on $p$-adic groups, our motivation comes from Jacquet's relative trace formula. A principal step in establishing such a formula is the proof of the corresponding fundamental lemma. In [7], Ye used the inversion formula for the Gelfand transform to prove the fundamental lemma with respect to the entire Hecke algebra for a relative trace formula for GL (3). Later Ye [8] generalized the inversion formula to GL $(n)$. We proved the fundamental lemmas with respect to the unit element for certain relative trace formulas for GSp (4) in [4]. We were inspired by Ye's work [7, 8, to consider the material in this article so that we could extend our fundamental lemmas in [4] to the entire Hecke algebra.

Received by the editors August 7, 2000 and, in revised form, February 26, 2001.

2000 Mathematics Subject Classification. Primary 22E35; Secondary 11F70, 11F72.

The research of Furusawa was partially supported by the Sumitomo Foundation and the Grantin-Aid for Scientific Research No. 10640028, the Ministry of Education, Science, Sports and Culture of Japan. 
Notation. Let $F$ be a non-archimedean local field. In Section 2 we assume that the residual characteristic of $F$ is different from two. Let $\mathcal{O}$ denote the ring of integers of $F, \varpi$ denote a local uniformizer, and $q$ denote the cardinality of the residue field $\mathcal{O} / \varpi \mathcal{O}$. By a character of a topological group, we always mean a continuous homomorphism to the group of complex numbers of absolute value 1 .

\section{Plancherel measure and the inversion formula FOR THE BESSEL TRANSFORM}

2.1. Bessel model. For $m \geq 2$, let $\mathrm{SO}(m, F)$ denote the split group of determinant one orthogonal matrices

$$
\mathrm{SO}(m, F)=\left\{g \in \mathrm{SL}(m, F) \mid(g x, g y)=(x, y) \text { for all } x, y \in F^{m}\right\}
$$

where $($,$) is the quadratic form (x, y)=\sum_{i=1}^{m} x_{i} y_{m+1-i}$.

Let $G=\mathrm{SO}(2 n+1, F)$, where $n \geq 2$, and let $U$ be the subgroup of $G$ consisting of upper triangular unipotent matrices whose center $3 \times 3$ block is the identity. Given $S=(a, b, c) \in \mathcal{O}^{3}$ such that $\operatorname{gcd}(a, b, c)=1$ and $b^{2}+2 a c \neq 0$, let us define a character $\theta_{S}$ of $U$ by the formula

$$
\theta_{S}(u)=\psi\left(u_{1,2}+u_{2,3}+\cdots+u_{n-2, n-1}+a u_{n-1, n}+b u_{n-1, n+1}+c u_{n-1, n+2}\right)
$$

where $\psi$ is a character of $F$ of conductor $\mathcal{O}$. Let $T$ be the connected component of the subgroup of $G$ consisting of matrices of the form

$$
\left(\begin{array}{ccc}
1_{n-1} & 0 & 0 \\
0 & g & 0 \\
0 & 0 & 1_{n-1}
\end{array}\right)
$$

which, acting by conjugation, stabilizes $\theta_{S}$. Then $T$ is a torus in $G$, which may be either split or non-split over $F$, depending on $S$.

When $T$ is split, we may and will suppose that $S=(1,0,1)$. Then $T$ consists of matrices of the form (1) where

$$
g=\left(\begin{array}{ccc}
a & 0 & 0 \\
0 & 1 & 0 \\
0 & 0 & a^{-1}
\end{array}\right), \quad a \in F^{\times} .
$$

Since $T$ normalizes $U$, we have a subgroup $R:=T U$ of $G$. Let $\lambda$ be a character of $T$ which is trivial on the maximal compact subgroup of $T$. Then we extend $\theta_{S}$ to a character of $R$ by

$$
\theta_{S}(t u)=\lambda(t) \theta_{S}(u)
$$

Let $\left(\pi, V_{\pi}\right)$ be an admissible representation of $G$. Then a Bessel functional on $\pi$ is a linear functional $H: V_{\pi} \rightarrow \mathbb{C}$ such that

$$
H(\pi(r) v)=\theta_{S}(r) H(v) \quad \text { for } r \in R \text { and } v \in V_{\pi} .
$$

As shown by Novodvorsky [6], if $\pi$ is irreducible then the dimension of the space of such functionals is at most one.

Let $P$ be the standard Borel subgroup of $G$, consisting of upper triangular matrices in $G$. Let $P=A N$ be its Levi decomposition where $A$ is the maximal torus of $G$ contained in $P$ and $N$ is the unipotent radical of $P$. Namely $A$ consists of diagonal matrices of the form

$$
\operatorname{diag}\left(a_{1}, a_{2}, \cdots, a_{n}, 1, a_{n}^{-1}, \cdots, a_{2}^{-1}, a_{1}^{-1}\right) .
$$


For a character $\chi$ of $A$, let $I(\chi)$ denote the induced representation $\operatorname{Ind}_{P}^{G}(\chi)$ defined as usual. We recall that $I(\chi)$ is the space of locally constant functions $\Phi: G \rightarrow \mathbb{C}$ which satisfy

$$
\Phi(\text { ang })=\delta_{P}(a)^{\frac{1}{2}} \chi(a) \Phi(g) \quad \text { for } a \in A, n \in N \text { and } g \in G,
$$

where $\delta_{P}$ denotes the modulus function for $P$. The action of $G$ is given by the right regular representation, i.e. $R_{g} \Phi(x)=\Phi(x g)$ for $g, x \in G$.

Let $K$ be the standard maximal compact subgroup $G(\mathcal{O})$ of $G$. In [1] it is shown that when $\chi$ is unramified, i.e. $\chi$ is trivial on $A \cap K$, there exists a Bessel functional $H_{\chi}: I(\chi) \rightarrow \mathbb{C}$ such that

$$
H_{\chi}\left(\varphi_{K}\right)=1
$$

where $\varphi_{K}$ denotes the $K$-fixed element in $I(\chi)$ with $\varphi_{K}(1)=1$. Then the normalized unramified Bessel function $B_{\chi}: G \rightarrow \mathbb{C}$ corresponding to $\chi$ is defined by

$$
B_{\chi}(g)=H_{\chi}\left(R_{g} \varphi_{K}\right)
$$

and satisfies the condition

$$
B_{\chi}(1)=1 \text { and } B_{\chi}(r g k)=\theta_{S}(r) B_{\chi}(g) \text { for } r \in R, g \in G \text { and } k \in K \text {. }
$$

2.2. Plancherel measure. We denote by $\mathcal{B}$ the space of functions $h: G \rightarrow \mathbb{C}$ satisfying:

$$
\begin{aligned}
& h(r g k)=\theta_{S}(r)^{-1} \cdot h(g) \text { for } r \in R, g \in G, \text { and } k \in K, \\
& |h| \text { is compactly supported on } R \backslash G .
\end{aligned}
$$

Let $\hat{A}$ be the group of unramified characters of $A$. Then for $h \in \mathcal{B}$, we define its Fourier transform $\hat{h}: \hat{A} \rightarrow \mathbb{C}$ by

$$
\hat{h}(\chi)=\int_{R \backslash G} h(g) B_{\chi}(g) d g
$$

where the measure on $R \backslash G$ is normalized so that $\int_{R \backslash R K} d g=1$.

Let us identify $\mathbf{m}=\left(m_{1}, m_{2}, \ldots, m_{n}\right) \in \mathbb{Z}^{n}$ with an element $d_{\mathbf{m}} \in A$ given by

$$
d_{\mathbf{m}}=\operatorname{diag}\left(\varpi^{m_{1}}, \varpi^{m_{2}}, \ldots, \varpi^{m_{n}}, 1, \varpi^{-m_{n}}, \ldots, \varpi^{-m_{2}}, \varpi^{-m_{1}}\right)
$$

and for each integer $i(1 \leq i \leq n)$, let $\mathbf{e}_{i}$ denote the $i$-th fundamental vector in $\mathbb{Z}^{n}$, i.e. the $i$-th entry of $\mathbf{e}_{i}$ is one and the other entries of $\mathbf{e}_{i}$ are all zero. For a character $\chi$ of $A$ and $\mathbf{m} \in \mathbb{Z}^{n}$, we write $\langle\chi, \mathbf{m}\rangle$ for $\chi\left(d_{\mathbf{m}}\right)$.

Let us define a measure $\nu$ on $\hat{A}$ by

$$
d \nu(\chi)=\frac{Q\left(q^{-1}\right)}{|W|} \cdot \frac{d \chi}{|C(\chi)|^{2}}
$$

where $|W|$ denotes the cardinality of the Weyl group $W$ of $G$,

$$
Q\left(q^{-1}\right)= \begin{cases}1+q^{-1}, & \text { when } T \text { is non-split } \\ 1-q^{-1}, & \text { when } T \text { is split }\end{cases}
$$




$$
\begin{aligned}
& C(\chi)=\prod_{\alpha>0} \frac{1}{1-\left\langle\chi, \boldsymbol{\alpha}^{\vee}\right\rangle} \\
& \quad \cdot \begin{cases}\prod_{i=1}^{n}\left(1-\left\langle\chi, \mathbf{e}_{i}\right\rangle q^{-\frac{1}{2}}\right)\left(1+\left\langle\chi, \mathbf{e}_{i}\right\rangle q^{-\frac{1}{2}}\right), & \text { when } T \text { is non-split, } \\
\prod_{i=1}^{n}\left(1-\left\langle\chi, \mathbf{e}_{i}\right\rangle \beta q^{-\frac{1}{2}}\right)\left(1-\left\langle\chi, \mathbf{e}_{i}\right\rangle \beta^{-1} q^{-\frac{1}{2}}\right), & \text { when } T \text { is split, }\end{cases}
\end{aligned}
$$

and, in ([6),$\alpha>0$ is taken over all positive roots of $G, \boldsymbol{\alpha}^{\vee}=\alpha^{\vee}(\varpi)$ where $\alpha^{\vee}$ denotes the coroot of $\alpha$ and $\beta=\lambda\left(d_{\mathbf{e}_{n}}\right)$. Also we normalize the Haar measure $d \chi$ so that $\int_{\hat{A}} d \chi=1$.

Theorem 1. For $h \in \mathcal{B}$, we have

$$
h(g)=\int_{\hat{A}} \hat{h}(\chi) \overline{B_{\chi}(g)} d \nu .
$$

Proof. Let us call a double coset $R g K$ relevant when

$$
\theta_{S}(r)=1 \quad \text { for } \quad r \in R \cap g K g^{-1} \text {. }
$$

Then it is clear that $h \in \mathcal{B}$ is supported on a finite union of relevant double cosets. We recall that a set of representatives for relevant double cosets is given in [1] as follows. We define a subset $\Lambda^{-}$of $\mathbb{Z}^{n}$ by

$$
\Lambda^{-}=\left\{\left(m_{1}, m_{2}, \ldots, m_{n}\right) \in \mathbb{Z}^{n} \mid m_{1} \geq m_{2} \geq \cdots \geq m_{n} \geq 0\right\}
$$

and let $n_{1}$ denote the unipotent matrix in $G$ given by

$$
n_{1}=\left(\begin{array}{ccccc}
1_{n-1} & 0 & 0 & 0 & 0 \\
0 & 1 & 1 & -\frac{1}{2} & 0 \\
0 & 0 & 1 & -1 & 0 \\
0 & 0 & 0 & 1 & 0 \\
0 & 0 & 0 & 0 & 1_{n-1}
\end{array}\right) .
$$

Then as a set of representatives for the relevant double cosets, we may take

$$
g_{\mathbf{m}}= \begin{cases}d_{\mathbf{m}}, & \text { when } T \text { is non-split } \\ n_{1} d_{\mathbf{m}}, & \text { when } T \text { is split }\end{cases}
$$

where $\mathbf{m} \in \Lambda^{-}$.

For each $\mathbf{m} \in \Lambda^{-}$, let us define $h_{\mathbf{m}} \in \mathcal{B}$ by

$$
h_{\mathbf{m}}(g)= \begin{cases}\theta_{S}(r)^{-1}, & \text { when } g=r g_{\mathbf{m}} k \text { for } r \in R \text { and } k \in K, \\ 0, & \text { otherwise. }\end{cases}
$$

Then the set $\left\{h_{\mathbf{m}} \mid \mathbf{m} \in \Lambda^{-}\right\}$is a basis for $\mathcal{B}$ and we have

$$
\hat{h}_{\mathbf{m}}(\chi)=\left(\int_{R \backslash R g_{\mathbf{m}} K} d g\right) \cdot B_{\chi}\left(g_{\mathbf{m}}\right) .
$$

Thus for (77), it is enough to show that for $\mathbf{m}, \mathbf{m}^{\prime} \in \Lambda^{-}$, we have

$$
\int_{\hat{A}} B_{\chi}\left(g_{\mathbf{m}}\right) \overline{B_{\chi}\left(g_{\mathbf{m}^{\prime}}\right)} d \nu=\left\{\begin{array}{cc}
\left(\int_{R \backslash R g_{\mathbf{m}} K} d g\right)^{-1}, & \text { when } \mathbf{m}=\mathbf{m}^{\prime}, \\
0, & \text { when } \mathbf{m} \neq \mathbf{m}^{\prime} .
\end{array}\right.
$$

Here it is easily seen that

$$
\int_{R \backslash R g_{\mathbf{m}} K} d g=\int_{R \cap g_{\mathbf{m}} K g_{\mathbf{m}}^{-1}} d r= \begin{cases}\delta_{B}\left(g_{\mathbf{m}}\right)^{-1}, & \text { when } m_{n}=0, \\ \delta_{B}\left(g_{\mathbf{m}}\right)^{-1} \cdot Q\left(q^{-1}\right), & \text { when } m_{n}>0 .\end{cases}
$$


We recall that by the explicit formula in [1, we have

$$
B_{\chi}\left(g_{\mathbf{m}}\right)=\frac{\delta_{P}\left(g_{\mathbf{m}}\right)^{\frac{1}{2}}}{Q\left(q^{-1}\right)} \sum_{w \in W} C(w \chi) \cdot w \chi\left(g_{\mathbf{m}}\right)^{-1} .
$$

Let $\alpha_{i}=\left\langle\chi, \mathbf{e}_{i}\right\rangle$. Then we note that in terms of its action on the parameters $\left(\alpha_{1}, \ldots, \alpha_{n}\right)$, the Weyl group $W$ is the group of transformations generated by

$$
\left(\alpha_{1}, \ldots, \alpha_{n-1}, \alpha_{n}\right) \mapsto\left(\alpha_{1}, \ldots, \alpha_{n-1}, \alpha_{n}^{-1}\right)
$$

and by the action of the symmetric group $S_{n}$ on $\left(\alpha_{1}, \ldots, \alpha_{n}\right)$. The cardinality of $W$ is $2^{n} n$ !.

Let

$$
\Delta(\chi)=\left\langle\chi,-\sum_{i=1}^{n}(n+1-i) \mathbf{e}_{i}\right\rangle \cdot \prod_{\alpha>0}\left(1-\left\langle\chi, \boldsymbol{\alpha}^{\vee}\right\rangle\right) .
$$

Then by Weyl's identity for $\operatorname{Sp}_{2 n}(\mathbb{C})$, we have

$$
\Delta(\chi)=(-1)^{n} \mathcal{A}\left(\alpha_{1}^{n} \alpha_{2}^{n-1} \cdots \alpha_{n}\right)
$$

where $\mathcal{A}$ denotes the alternator $\sum_{w \in W} \operatorname{sgn}(w) w$ in the group algebra $\mathbb{C}[W]$. We recall that $\operatorname{sgn}(w)=(-1)^{\text {length }(w)}$ for $w \in W$. Hence by (10), we have

$$
\Delta(w \chi)=\operatorname{sgn}(w) \cdot \Delta(\chi) \quad \text { for } w \in W
$$

and we may write (9) as

$$
B_{\chi}\left(g_{\mathbf{m}}\right)=\frac{\delta_{P}\left(g_{\mathbf{m}}\right)^{\frac{1}{2}}}{Q\left(q^{-1}\right) \Delta(\chi)} \cdot \mathcal{A}\left[\gamma(\chi) \cdot \chi\left(g_{\mathbf{m}}\right)^{-1}\right]
$$

where

$$
\begin{aligned}
& \gamma(\chi)=\left\langle\chi,-\sum_{i=1}^{n}(n+1-i) \mathbf{e}_{i}\right\rangle \\
& \cdot \begin{cases}\prod_{i=1}^{n}\left(1-\left\langle\chi, \mathbf{e}_{i}\right\rangle q^{-\frac{1}{2}}\right)\left(1+\left\langle\chi, \mathbf{e}_{i}\right\rangle q^{-\frac{1}{2}}\right), & \text { when } T \text { is non-split, } \\
\prod_{i=1}^{n}\left(1-\left\langle\chi, \mathbf{e}_{i}\right\rangle \beta q^{-\frac{1}{2}}\right)\left(1-\left\langle\chi, \mathbf{e}_{i}\right\rangle \beta^{-1} q^{-\frac{1}{2}}\right), & \text { when } T \text { is split. }\end{cases}
\end{aligned}
$$

Here we note that $|\gamma(\chi)|^{2}=\gamma(\chi) \gamma\left(\chi^{-1}\right)$ is $W$-invariant from (11). Hence

$$
\frac{1}{|C(\chi)|^{2}} B_{\chi}\left(g_{\mathbf{m}}\right)=\frac{\delta_{P}\left(g_{\mathbf{m}}\right)^{\frac{1}{2}} \Delta\left(\chi^{-1}\right)}{Q\left(q^{-1}\right)} \cdot \mathcal{A}\left[\gamma\left(\chi^{-1}\right)^{-1} \cdot \chi\left(g_{\mathbf{m}}\right)^{-1}\right]
$$

and we have

$$
\begin{aligned}
\int_{\hat{A}} & B_{\chi}\left(g_{\mathbf{m}}\right) \overline{B_{\chi}\left(g_{\mathbf{m}^{\prime}}\right)} d \nu \\
& =\frac{\delta_{B}\left(g_{\mathbf{m}} g_{\mathbf{m}^{\prime}}\right)^{\frac{1}{2}}}{|W| \cdot Q\left(q^{-1}\right)} \int_{\hat{A}} \mathcal{A}\left[\gamma\left(\chi^{-1}\right)^{-1} \cdot \chi\left(g_{\mathbf{m}}\right)^{-1}\right] \mathcal{A}\left[\gamma\left(\chi^{-1}\right) \cdot \chi\left(g_{\mathbf{m}^{\prime}}\right)\right] d \chi .
\end{aligned}
$$

Here we have

$$
\begin{aligned}
\mathcal{A}\left[\gamma\left(\chi^{-1}\right)^{-1} \cdot \chi\left(g_{\mathbf{m}}\right)^{-1}\right] \cdot \mathcal{A}[\gamma & \left.\left(\chi^{-1}\right) \cdot \chi\left(g_{\mathbf{m}^{\prime}}\right)\right] \\
& =\sum_{w, \sigma \in W} \operatorname{sgn}(\sigma)\left\langle w \chi,-\mathbf{m}+\sigma \mathbf{m}^{\prime}\right\rangle \cdot A(w \chi, \sigma)
\end{aligned}
$$


where

$$
\begin{aligned}
A(w \chi, \sigma)= & \prod_{i=1}^{n}\left\langle w \chi,-(n+1-i)\left(\mathbf{e}_{i}-\sigma \mathbf{e}_{i}\right)\right\rangle \\
& \cdot \begin{cases}\frac{\left(1-\left\langle w \chi,-\sigma \mathbf{e}_{i}\right\rangle q^{-\frac{1}{2}}\right)\left(1+\left\langle w \chi,-\sigma \mathbf{e}_{i}\right\rangle q^{-\frac{1}{2}}\right)}{\left(1-\left\langle w \chi,-\mathbf{e}_{i}\right\rangle q^{-\frac{1}{2}}\right)\left(1+\left\langle w \chi,-\mathbf{e}_{i}\right\rangle q^{-\frac{1}{2}}\right)}, & \text { when } T \text { is non-split, } \\
\frac{\left(1-\left\langle w \chi,-\sigma \mathbf{e}_{i}\right\rangle \beta q^{-\frac{1}{2}}\right)\left(1-\left\langle w \chi,-\sigma \mathbf{e}_{i}\right\rangle \beta^{-1} q^{-\frac{1}{2}}\right)}{\left(1-\left\langle w \chi,-\mathbf{e}_{i}\right\rangle \beta q^{-\frac{1}{2}}\right)\left(1-\left\langle w \chi,-\mathbf{e}_{i}\right\rangle \beta^{-1} q^{-\frac{1}{2}}\right)}, & \text { when } T \text { is split. }\end{cases}
\end{aligned}
$$

Since the rest of the proofs are identical, we consider only the split case here. By the Schur orthogonality, we have

$$
\begin{gathered}
\quad \frac{1}{|W|} \sum_{w, \sigma \in W} \operatorname{sgn}(\sigma) \int_{\hat{A}}\left\langle w \chi,-\mathbf{m}+\sigma \mathbf{m}^{\prime}\right\rangle \cdot A(w \chi, \sigma) d \chi \\
=\sum_{\sigma \in W} \operatorname{sgn}(\sigma) \sum_{i=1}^{n} \sum_{\substack{k_{i}^{(1)}, k_{i}^{(2)} \geq 0 \\
\ell_{i}^{(1)}, \ell_{i}^{(2)} \in\{0,1\}}} \sum_{\beta^{\sum_{i=1}^{n}\left(\ell_{i}^{(1)}-\ell_{i}^{(2)}+k_{i}^{(1)}-k_{i}^{(2)}\right)} q^{-\frac{1}{2} \sum_{i=1}^{n}\left(\ell_{i}^{(1)}+\ell_{i}^{(2)}+k_{i}^{(1)}+k_{i}^{(2)}\right)}}^{(-1)^{\sum_{i=1}^{n}\left(\ell_{i}^{(1)}+\ell_{i}^{(2)}\right)}} \\
\cdot \delta\left[\mathbf{m}-\sigma \mathbf{m}^{\prime}+\sum_{i=1}^{n}\left\{\left(k_{i}^{(1)}+k_{i}^{(2)}\right) \mathbf{e}_{i}+(n+1-i)\left(\mathbf{e}_{i}-\sigma \mathbf{e}_{i}\right)+\left(\ell_{i}^{(1)}+\ell_{i}^{(2)}\right) \sigma \mathbf{e}_{i}\right\}\right] .
\end{gathered}
$$

Here for $\mathbf{t} \in \mathbb{Z}^{n}$,

$$
\delta(\mathbf{t})= \begin{cases}1, & \text { when } \mathbf{t}=0, \\ 0, & \text { otherwise }\end{cases}
$$

It is clear from (13) that the integral (12) is real valued. Hence it is symmetric with respect to $\mathbf{m} \leftrightarrow \mathbf{m}^{\prime}$. Thus we may assume that $\mathbf{m} \geq \mathbf{m}^{\prime}$ with respect to the lexicographic ordering of $\mathbb{Z}^{n}$. Then it is clear that the contribution to (13) from $\sigma=1$ is $\delta\left(\mathbf{m}-\mathbf{m}^{\prime}\right)$. Suppose that $\sigma \neq 1$. Let $r$ be the smallest integer $1 \leq r \leq n$ such that $\sigma \mathbf{e}_{r} \neq \mathbf{e}_{r}$. Then we have $\sigma \mathbf{e}_{s}= \pm \mathbf{e}_{r}$ where $r \leq s \leq n$. If we have a non-zero contribution to (13) from a term corresponding to $\sigma$, it is clear that we have

$$
m_{i}-m_{i}^{\prime}=k_{i}^{(1)}=k_{i}^{(2)}=\ell_{i}^{(1)}=\ell_{i}^{(2)}=0 \quad \text { for } 1 \leq i<r .
$$

The $r$-th entry of the vector inside the bracket for $\delta$ in (13) is given by

$$
\begin{gathered}
\left(m_{r}-m_{s}^{\prime}\right)+\left(k_{r}^{(1)}+k_{r}^{(2)}\right)+(s-r)+\left(\ell_{s}^{(1)}+\ell_{s}^{(2)}\right) \quad \text { when } \sigma \mathbf{e}_{s}=\mathbf{e}_{r}, \\
\left(m_{r}+m_{s}^{\prime}\right)+\left(k_{r}^{(1)}+k_{r}^{(2)}\right)+(2 n+2-r-s)-\left(\ell_{s}^{(1)}+\ell_{s}^{(2)}\right) \\
\text { when } \sigma \mathbf{e}_{s}=-\mathbf{e}_{r} .
\end{gathered}
$$

In the first case, we have $r<s$ and we also have $m_{r} \geq m_{r}^{\prime} \geq m_{s}^{\prime}$ since $\mathbf{m} \geq \mathbf{m}^{\prime}$ and $\mathbf{m}^{\prime} \in \Lambda^{-}$. Hence the $r$-th entry (14) is strictly positive and we have no contribution. In the second case we note that $2 n+2-r-s \geq 2$. Hence the $r$-th entry (15) is nonnegative and it becomes zero only when $r=s=n, m_{n}=m_{n}^{\prime}=k_{n}^{(1)}=k_{n}^{(2)}=0$, 
and $\ell_{n}^{(1)}=\ell_{n}^{(2)}=1$. Thus we have

$$
\begin{aligned}
& \frac{1}{|W|} \sum_{w, \sigma \in W} \operatorname{sgn}(\sigma) \int_{\hat{A}}\left\langle w \chi,-\mathbf{m}+\sigma \mathbf{m}^{\prime}\right\rangle \cdot A(w \chi, \sigma) d \chi \\
& \quad= \begin{cases}1, & \text { when } \mathbf{m}=\mathbf{m}^{\prime} \text { and } m_{n}>0 \\
1-q^{-1}, & \text { when } \mathbf{m}=\mathbf{m}^{\prime} \text { and } m_{n}=0, \\
0, & \text { otherwise. }\end{cases}
\end{aligned}
$$

Hence (8) holds.

2.3. Inversion formula for the Bessel transform. Let $\mathcal{H}(=\mathcal{H}(G, K))$ be the Hecke algebra of $G$ with respect to $K$. Namely $\mathcal{H}$ is the set of compactly supported functions on $G$ which are $K$-bi-invariant:

$$
f: G \rightarrow \mathbb{C} \text { satisfying } f\left(k_{1} x k_{2}\right)=f(x) \text { for all } k_{1}, k_{2} \in K .
$$

Then for $f \in \mathcal{H}$, we define its Bessel transform $\Psi_{f}: G \rightarrow \mathbb{C}$ by

$$
\Psi_{f}(g)=\int_{R} f(g r) \theta_{S}(r)^{-1} d r
$$

Theorem 2. For $\mathbf{m} \in \Lambda^{-}$, we have

$$
\begin{aligned}
\Psi_{f}\left(g_{\mathbf{m}}^{-1}\right)=\delta_{P}\left(d_{\mathbf{m}}\right)^{\frac{1}{2}} \sum_{\alpha>0} \sum_{m_{\alpha} \in\{0,1\}} \sum_{1 \leq i \leq n} \sum_{\ell_{i} \geq 0} \\
(-1)^{\sum_{\alpha>0} m_{\alpha}} q^{-\sum_{i=1}^{n} \ell_{i}} \cdot S f\left[\mathbf{m}+\sum_{\alpha>0} m_{\alpha} \boldsymbol{\alpha}^{\vee}+2 \sum_{i=1}^{n} \ell_{i} \mathbf{e}_{i}\right]
\end{aligned}
$$

when $T$ is non-split, and

$$
\begin{aligned}
& \Psi_{f}\left(g_{\mathbf{m}}^{-1}\right)=\delta_{P}\left(d_{\mathbf{m}}\right)^{\frac{1}{2}} \sum_{\alpha>0} \sum_{m_{\alpha} \in\{0,1\}} \sum_{1 \leq i \leq n} \sum_{\ell_{i}^{(1)}, \ell_{i}^{(2)} \geq 0} \\
& (-1)^{\sum_{\alpha>0} m_{\alpha}} q^{-\frac{1}{2} \sum_{i=1}^{n}\left(\ell_{i}^{(1)}+\ell_{i}^{(2)}\right)} \beta^{\sum_{i=1}^{n}\left(\ell_{i}^{(1)}-\ell_{i}^{(2)}\right)} \\
& \cdot S f\left[\mathbf{m}+\sum_{\alpha>0} m_{\alpha} \boldsymbol{\alpha}^{\vee}+\sum_{i=1}^{n}\left(\ell_{i}^{(1)}+\ell_{i}^{(2)}\right) \mathbf{e}_{i}\right]
\end{aligned}
$$

when $T$ is split, where $S f$ denotes the Satake transform of $f$ defined by

$$
S f(a)=\delta_{P}(a)^{\frac{1}{2}} \int_{A} f(a n) d n, \quad a \in A,
$$

and we write $S f\left(\mathbf{m}^{\prime}\right)$ for $S f\left(d_{\mathbf{m}^{\prime}}\right)$ when $\mathbf{m}^{\prime} \in \mathbb{Z}^{n}$. Here we normalize the measures so that

$$
\int_{R \cap K} d r=\int_{N \cap K} d n=1
$$

We note that the summations in (17) and (18) are indeed finite since $S f$ is compactly supported.

Proof. For $f \in \mathcal{H}$, let us define $h: G \rightarrow \mathbb{C}$ by $h(g)=\Psi_{f}\left(g^{-1}\right)$. It is clear that $h \in \mathcal{B}$ and we have

$$
\hat{h}(\chi)=\int_{R \backslash G} \Psi_{f}\left(g^{-1}\right) B_{\chi}(g) d g=\int_{G} f\left(g^{-1}\right) B_{\chi}(g) d g .
$$


Furthermore we note that the function

$$
G \ni g \mapsto \int_{K} B_{\chi}(k g) d k \in \mathbb{C}
$$

coincides with $\Gamma_{\chi}$, the spherical function on $G$ associated to $\chi$, and we have

$\hat{h}(\chi)=\int_{K} \int_{G} f\left(g^{-1} k\right) B_{\chi}(g) d g d k=\int_{G} f\left(g^{-1}\right) \Gamma_{\chi}(g) d g=\int_{G} f(g) \Gamma_{\chi}\left(g^{-1}\right) d g$.

Since $\Gamma_{\chi}\left(g^{-1}\right)=\Gamma_{\chi^{-1}}(g)$, we have

$$
\hat{h}(\chi)=\int_{G} f(g) \Gamma_{\chi^{-1}}(g) d g=\int_{A} S f(a) \chi^{-1}(a) d a .
$$

Thus by Theorem 1 we have

$$
\Psi_{f}\left(g_{\mathbf{m}}^{-1}\right)=\int_{\hat{A}} \int_{A} S f(a) \chi^{-1}(a) \overline{B_{\chi}\left(g_{\mathbf{m}}\right)} d a d \nu
$$

Here $S f: A \rightarrow \mathbb{C}$ is compactly supported and it is easily seen that

$$
\int_{\hat{A}}\left|B_{\chi}\left(g_{\mathbf{m}}\right)\right| d \nu<+\infty .
$$

Thus we may change the order of integration in (201) and we have

$$
\Psi_{f}\left(g_{\mathbf{m}}^{-1}\right)=\sum_{\mathbf{m}^{\prime} \in \mathbb{Z}^{n}} S f\left(\mathbf{m}^{\prime}\right) K\left(\mathbf{m}, \mathbf{m}^{\prime}\right)
$$

where

$$
K\left(\mathbf{m}, \mathbf{m}^{\prime}\right)=\int_{\hat{A}} \chi^{-1}\left(d_{\mathbf{m}^{\prime}}\right) \overline{B_{\chi}\left(g_{\mathbf{m}}\right)} d \nu=\frac{\delta_{P}\left(d_{\mathbf{m}}\right)^{\frac{1}{2}}}{|W|} \sum_{w \in W} K_{w}\left(\mathbf{m}, \mathbf{m}^{\prime}\right)
$$

and

$$
K_{w}\left(\mathbf{m}, \mathbf{m}^{\prime}\right)=\int_{\hat{A}} C(w \chi)^{-1} \cdot\left\langle w \chi,-w \mathbf{m}^{\prime}+\mathbf{m}\right\rangle d \chi
$$

since $|C(\chi)|^{2}=C(\chi) C\left(\chi^{-1}\right)$ is $W$-invariant.

In the split case, (21) becomes

$$
\begin{aligned}
& K_{w}\left(\mathbf{m}, \mathbf{m}^{\prime}\right)= \sum_{m_{\alpha} \in\{0,1\} \text { for } \alpha>0 \quad \ell_{i}^{(1)}, \ell_{i}^{(2)} \geq 0 \text { for } 1 \leq i \leq n} \\
&(-1)^{\sum_{\alpha>0} m_{\alpha}} q^{-\frac{1}{2} \sum_{i=1}^{n}\left(\ell_{i}^{(1)}+\ell_{i}^{(2)}\right)} \beta^{\sum_{i=1}^{n}\left(\ell_{i}^{(1)}-\ell_{i}^{(2)}\right)} \\
& \cdot \delta\left[-w \mathbf{m}^{\prime}+\mathbf{m}+\sum_{\alpha>0} m_{\alpha} \boldsymbol{\alpha}^{\vee}+\sum_{i=1}^{n}\left(\ell_{i}^{(1)}+\ell_{i}^{(2)}\right) \mathbf{e}_{i}\right] .
\end{aligned}
$$

Since we have $S f\left(\mathbf{m}^{\prime}\right)=S f\left(w \mathbf{m}^{\prime}\right)$ for $\mathbf{m}^{\prime} \in \mathbb{Z}^{n}$ and $w \in W$, we obtain (18) by rearranging the terms. The proof for the non-split case is similar. 
2.4. GSp (4) case. In view of the isomorphism $\mathrm{SO}(5, F) \simeq \operatorname{PGSp}(4, F)$, the theorems above imply the existence of the Plancherel measure and the inversion formula for the Bessel transform on $\operatorname{GSp}(4, F)$. We shall state them here, despite some redundancy, because of its crucial use in our concurrent work to generalize the fundamental lemmas for the unit element in [4 to the whole Hecke algebra.

We use the following notation in this subsection. Consider the group

$$
G=\left\{g \in \mathrm{GL}(4, F) \mid{ }^{t} g J g=\mu(g) J, \mu(g) \in \mathrm{GL}(1, F)\right\} \quad \text { where } J=\left(\begin{array}{cc}
0 & 1_{2} \\
-1_{2} & 0
\end{array}\right) .
$$

Let $S=\left(\begin{array}{cc}a & b / 2 \\ b / 2 & c\end{array}\right)$ such that $a, b, c \in \mathcal{O}, \operatorname{gcd}(a, b, c)=1$ and $\operatorname{det} S \neq 0$. Let $U$ be the unipotent radical of the upper Siegel parabolic subgroup

$$
U=\left\{u=\left(\begin{array}{cc}
1_{2} & X \\
0 & 1_{2}
\end{array}\right) \mid X={ }^{t} X\right\}
$$

and let $\theta_{S}$ be the character of $U$ given by $\theta_{S}(u)=\psi(\operatorname{tr}(S X))$ where $\psi$ is a character of $F$ of conductor $\mathcal{O}$. Let $T$ be the torus in $G$ defined by

$$
T=\left\{\left(\begin{array}{cc}
h & 0 \\
0 & \operatorname{det}(h) \cdot{ }^{t} h^{-1}
\end{array}\right) \mid{ }^{t} h S h=\operatorname{det} h \cdot S\right\} .
$$

Then $T$ stabilizes $\theta_{S}$ by conjugation. For a character $\lambda$ of $T$ which is trivial on the maximal compact subgroup of $T$, we extend $\theta_{S}$ to a character of the group $R:=T U$ by $\theta_{S}(t u)=\lambda(t) \theta_{S}(u)$.

When $T$ is split, we suppose that $S=\left(\begin{array}{cc}0 & 1 / 2 \\ 1 / 2 & 0\end{array}\right)$ without loss of generality and then we have

$$
T=\{\operatorname{diag}(a, b, b, a) \mid a, b \in \mathrm{GL}(1, F)\} .
$$

Let $K$ denote the standard maximal compact subgroup $\operatorname{GSp}(4, \mathcal{O})$ of $G$. Let $A$ denote the standard split maximal torus of $G$, consisting of diagonal matrices of the form

$$
\operatorname{diag}(a, b, c, d) \quad \text { where } a c=b d .
$$

Let

$$
\Lambda=\left\{\left(m_{1}, m_{2}, m_{3}, m_{4}\right) \in \mathbb{Z}^{4} \mid m_{1}+m_{3}=m_{2}+m_{4}\right\} .
$$

Then for $\mathbf{m}=\left(m_{1}, m_{2}, m_{3}, m_{4}\right) \in \Lambda$, we define $a_{\mathbf{m}} \in A$ by

$$
a_{\mathbf{m}}=\operatorname{diag}\left(\varpi^{m_{1}}, \varpi^{m_{2}}, \varpi^{m_{3}}, \varpi^{m_{4}}\right) .
$$

For a character $\chi$ of $A$, we write $\langle\chi, \mathbf{m}\rangle$ for $\chi\left(a_{\mathbf{m}}\right)$. Let $N$ denote the subgroup of $G$ defined by

$$
N=\left\{\left(\begin{array}{cccc}
1 & x & 0 & 0 \\
0 & 1 & 0 & 0 \\
0 & 0 & 1 & 0 \\
0 & 0 & -x & 1
\end{array}\right)\left(\begin{array}{cccc}
1 & 0 & r & s \\
0 & 1 & s & t \\
0 & 0 & 1 & 0 \\
0 & 0 & 0 & 1
\end{array}\right) \mid x, r, s, t \in F\right\}
$$

Then $P:=A N$ is the standard Borel subgroup of $G$.

Let $\hat{A}$ denote the group of unramified characters of $A$. Let us denote by $\omega$ the restriction of $\lambda$ to $Z$, the center of $G$, and let

$$
\hat{A}(\omega)=\left\{\chi \in \hat{A}|\chi|_{Z}=\omega\right\} \text {. }
$$


Let $\rho$ be an unramified character of $F^{\times}$such that $\rho^{2}=\omega$ and let us define a character $\chi_{0} \in \hat{A}(\omega)$ by

$$
\chi_{0}(a)=\rho(\mu(a)) \quad \text { where } \mu \text { denotes the similitude. }
$$

Then we have $\hat{A}(\omega)=\chi_{0} \cdot \hat{A}(1)$ and we note that $\hat{A}(1)$ is a group isomorphic to

$$
\left\{\left(z_{1}, z_{2}\right) \in \mathbb{C}^{\times} \times \mathbb{C}^{\times}|| z_{1}|=| z_{2} \mid=1\right\} .
$$

Let $d \tau$ denote the Haar measure on $\hat{A}(1)$ normalized so that $\int_{\hat{A}(1)} d \tau=1$.

For $\chi \in \hat{A}(\omega)$, let $B_{\chi}: G \rightarrow \mathbb{C}$ denote the corresponding normalized unramified Bessel function. Thus we have

$$
B_{\chi}(1)=1 \text { and } B_{\chi}(r g k)=\theta_{S}(r) B_{\chi}(g) \text { for } r \in R, g \in G \text { and } k \in K \text {. }
$$

As in the $\mathrm{SO}(2 n+1)$ case, let us denote by $\mathcal{B}$ the space of functions $h: G \rightarrow \mathbb{C}$ satisfying (2) and (3). For $h \in \mathcal{B}$, we define its Fourier transform $\hat{h}: \hat{A}(\omega) \rightarrow \mathbb{C}$ by (4).

Let us define a measure $\nu$ on $\hat{A}(\omega)=\chi_{0} \cdot \hat{A}(1)$ by

$$
d \nu(\chi)=\frac{Q\left(q^{-1}\right)}{|W|} \cdot \frac{d \tau}{|C(\tau)|^{2}}, \quad \chi=\chi_{0} \cdot \tau
$$

where $|W|$ denotes the cardinality of the Weyl group $W$ of $G, Q\left(q^{-1}\right)$ is defined as in (5), and,

$$
\begin{aligned}
C(\tau) & =\prod_{\alpha>0} \frac{1}{1-\left\langle\tau, \boldsymbol{\alpha}^{\vee}\right\rangle} \\
& \cdot \begin{cases}\prod_{i=1,2}\left(1-\left\langle\tau, \mathbf{e}_{i}^{\prime}\right\rangle q^{-\frac{1}{2}}\right)\left(1+\left\langle\tau, \mathbf{e}_{i}^{\prime}\right\rangle q^{-\frac{1}{2}}\right), & \text { when } T \text { is non-split, } \\
\prod_{i=1,2}\left(1-\left\langle\tau, \mathbf{e}_{i}^{\prime}\right\rangle \beta q^{-\frac{1}{2}}\right)\left(1-\left\langle\tau, \mathbf{e}_{i}^{\prime}\right\rangle \beta^{-1} q^{-\frac{1}{2}}\right), & \text { when } T \text { is split, }\end{cases}
\end{aligned}
$$

where

$$
\mathbf{e}_{1}^{\prime}=(1,1,0,0), \quad \mathbf{e}_{2}^{\prime}=(0,1,1,0), \quad \beta=\lambda[\operatorname{diag}(\varpi, \varpi, 1,1)] \cdot \rho(\varpi)^{-1} .
$$

For $k, \ell \in \mathbb{Z}$, let $\mathbf{m}(k, \ell)=(k+2 \ell, k+\ell, 0, \ell) \in \Lambda$ and let $n_{1}$ denote the unipotent matrix in $G$ defined by

$$
n_{1}=\left(\begin{array}{cccc}
1 & 1 & 0 & 0 \\
0 & 1 & 0 & 0 \\
0 & 0 & 1 & 0 \\
0 & 0 & -1 & 1
\end{array}\right)
$$

Then as a set of representatives for relevant double cosets in $R \backslash G / K$, we may take

$$
b_{k, \ell}= \begin{cases}a_{\mathbf{m}(k, \ell)}, & \text { when } T \text { is non-split, } \\ n_{1} a_{\mathbf{m}(k, \ell)}, & \text { when } T \text { is split, }\end{cases}
$$

with $k, \ell \geq 0$. We recall that by the explicit formula in [1], we have

$$
B_{\chi}\left(b_{k, \ell}\right)=\frac{\chi_{0}\left(b_{k, \ell}\right) \delta_{P}\left(b_{k, \ell}\right)^{\frac{1}{2}}}{Q\left(q^{-1}\right)} \sum_{w \in W} C(w \tau) \cdot w \tau\left(b_{k, \ell}\right)^{-1} \quad \text { where } \chi=\chi_{0} \cdot \tau .
$$

Then from Theorem 1 , we have 
Corollary 1. For $h \in \mathcal{B}$, we have

$$
h(g)=\int_{\hat{A}(\omega)} \hat{h}(\chi) \overline{B_{\chi}(g)} d \nu .
$$

Let us consider the inversion formula. For an element $f$ in the Hecke algebra $\mathcal{H}(=\mathcal{H}(G, K))$, the Bessel transform $\Psi_{f}: G \rightarrow \mathbb{C}$ of $f$ is defined by (16).

Corollary 2. For $k, \ell \geq 0$, we have

$$
\begin{aligned}
\Psi_{f}\left(b_{k, \ell}^{-1}\right)=\chi_{0}\left(b_{k, \ell}\right)^{-2} \delta_{P}\left(b_{k, \ell}\right)^{\frac{1}{2}} & \sum_{\alpha>0} \sum_{m_{\alpha} \in\{0,1\}} \sum_{i=1,2} \sum_{\ell_{i} \geq 0} \sum_{n \in \mathbb{Z}} \\
(-1)^{\sum_{\alpha>0} m_{\alpha}} q^{-\sum_{i=1}^{2} \ell_{i}} \omega(\varpi)^{-n-\sum_{i=1,2} \ell_{i}} & \\
\cdot S f & {\left[\mathbf{m}(k, \ell)+\sum_{\alpha>0} m_{\alpha} \boldsymbol{\alpha}^{\vee}+2 \sum_{i=1}^{2} \ell_{i} \mathbf{e}_{i}^{\prime}+n \mathbf{z}\right] }
\end{aligned}
$$

when $T$ is non-split, and,

$$
\begin{gathered}
\Psi_{f}\left(b_{k, \ell}^{-1}\right)=\chi_{0}\left(b_{k, \ell}\right)^{-2} \delta_{P}\left(b_{k, \ell}\right)^{\frac{1}{2}} \sum_{\alpha>0} \sum_{m_{\alpha} \in\{0,1\}} \sum_{i=1,2} \sum_{\ell_{i}^{(1)}, \ell_{i}^{(2)} \geq 0} \sum_{n \in \mathbb{Z}} \\
(-1)^{\sum_{\alpha>0} m_{\alpha}} q^{-\frac{1}{2} \sum_{i=1}^{2}\left(\ell_{i}^{(1)}+\ell_{i}^{(2)}\right)} \omega(\varpi)^{-n-\sum_{i, j=1,2} \ell_{i}^{(j)}} \prod_{j=1}^{2} \beta_{j}^{\ell_{1}^{(j)}+\ell_{2}^{(j)}} \\
\cdot S f\left[\mathbf{m}(k, \ell)+\sum_{\alpha>0} m_{\alpha} \boldsymbol{\alpha}^{\vee}+\sum_{i=1}^{2}\left(\ell_{i}^{(1)}+\ell_{i}^{(2)}\right) \mathbf{e}_{i}^{\prime}+n \mathbf{z}\right]
\end{gathered}
$$

when $T$ is split. Here $S f$ denotes the Satake transform of $f$ defined by (19), $S f(\mathbf{n})=S f\left(a_{\mathbf{n}}\right)$ for $\mathbf{n} \in \Lambda$, and,

$$
\mathbf{z}=(1,1,1,1) \in \Lambda, \quad \beta_{1}=\lambda[\operatorname{diag}(\varpi, \varpi, 1,1)], \quad \beta_{2}=\lambda[\operatorname{diag}(1, \varpi, \varpi, 1)] .
$$

Proof. By an argument similar to the one in the proof of Theorem 2 we have

$$
\Psi_{f}\left(b_{k, \ell}^{-1}\right)=\sum_{\mathbf{m}^{\prime} \in \Lambda} S f\left(\mathbf{m}^{\prime}\right) K\left(\mathbf{m}(k, \ell), \mathbf{m}^{\prime}\right)
$$

where

$K\left(\mathbf{m}(k, \ell), \mathbf{m}^{\prime}\right)=\int_{\hat{A}(\omega)} \chi\left(a_{\mathbf{m}^{\prime}}\right)^{-1} \overline{B_{\chi}\left(b_{k, \ell}\right)} d \nu=\frac{\delta_{P}\left(b_{k, \ell}\right)^{\frac{1}{2}}}{|W|} \sum_{w \in W} K_{w}\left(\mathbf{m}(k, \ell), \mathbf{m}^{\prime}\right)$

and

$$
\begin{aligned}
& K_{w}\left(\mathbf{m}(k, \ell), \mathbf{m}^{\prime}\right)=\left\langle\chi_{0},-\mathbf{m}^{\prime}-\mathbf{m}(k, \ell)\right\rangle \\
& \cdot \int_{\hat{A}(1)} C(w \tau)^{-1} \cdot\left\langle w \tau,-w \mathbf{m}^{\prime}+\mathbf{m}(k, \ell)\right\rangle d \tau .
\end{aligned}
$$


In the split case we have

$$
\begin{aligned}
& K_{w}\left(\mathbf{m}(k, \ell), \mathbf{m}^{\prime}\right)=\left\langle\chi_{0},-\mathbf{m}^{\prime}-\mathbf{m}(k, \ell)\right\rangle \\
& \sum_{m_{\alpha} \in\{0,1\} \text { for } \alpha>0} \sum_{\ell_{i}^{(1)}, \ell_{i}^{(2)} \geq 0 \text { for } i=1,2} \sum_{n \in \mathbb{Z}} \\
& (-1)^{\sum_{\alpha>0} m_{\alpha}} q^{-\frac{1}{2} \sum_{i=1,2}\left(\ell_{i}^{(1)}+\ell_{i}^{(2)}\right)} \beta^{\sum_{i=1,2}\left(\ell_{i}^{(1)}-\ell_{i}^{(2)}\right)} \\
& \cdot \delta\left[-w \mathbf{m}^{\prime}+\mathbf{m}(k, \ell)+\sum_{\alpha>0} m_{\alpha} \boldsymbol{\alpha}^{\vee}+\sum_{i=1,2}\left(\ell_{i}^{(1)}+\ell_{i}^{(2)}\right) \mathbf{e}_{i}^{\prime}+n \mathbf{z}\right] .
\end{aligned}
$$

Here we recall that $\delta(\mathbf{n})=1$ when $\mathbf{n}=0, \delta(\mathbf{n})=0$ otherwise, for $\mathbf{n} \in \Lambda$.

The rest is clear and the non-split case is similar.

\section{Plancherel measure and the inversion formula FOR THE GELFAND TRANSFORM}

Our method for the construction of the Plancherel measure and the derivation of the inversion formula seems susceptible of generalizations to other unique models. In this section we shall show that this is indeed the case for the Whittaker model.

We use the notation consistent with that of [2, 3] as much as possible in this section to facilitate reference to them. Let $F$ be a non-archimedean local field and $\mathcal{O}$ be its ring of integers. Algebraic groups over $F$ will be written in boldface and their corresponding groups of $F$-rational points will be written in ordinary type as in $[2,3$. Let $\mathbf{G}$ be a connected reductive algebraic group defined over $F$. We assume that $\mathbf{G}$ is split over $F$. We take $K=\mathbf{G}(\mathcal{O})$ to be a maximal compact subgroup of $G=\mathbf{G}(F)$. Let $\mathbf{A}$ be a maximal split torus of $\mathbf{G}$ and let $\Sigma$ denote the roots of $\mathbf{G}$ with respect to $\mathbf{A}$. Let $\mathbf{P}=\mathbf{A N}$ be a minimal parabolic subgroup of $\mathbf{G}$ containing $\mathbf{A}$ where $\mathbf{N}$ is the unipotent radical of $\mathbf{P}$. Let

$\Sigma^{+}=$positive roots with respect to the choice of $\mathbf{P}, \quad \Delta=$ simple roots,

$$
W=\text { Weyl group of } \Sigma, \quad w_{\ell}=\text { longest element in } W .
$$

A character of $A$ is unramified if its restriction to $\mathbf{A}(\mathcal{O})=K \cap A$ is trivial. For a character $\chi$ of $A$, let $I(\chi)$ denote the induced representation $\operatorname{Ind}_{P}^{G}(\chi)$ defined as usual as in the previous section.

3.1. Whittaker model. Let $\psi$ be a principal character of $N$, i.e. $\psi$ is non-trivial on each root subgroup of $N$ corresponding to the simple roots. Suppose also that $\psi$ is unramified (see [3, p.219] for the definition of an unramified character of $N$ ). Let $\chi$ be an unramified character of $A$. Then it is shown in $[3]$ that there exists a unique linear functional $\Omega_{\chi}: I(\chi) \rightarrow \mathbb{C}$ such that

1. $\Omega_{\chi}\left(R_{n} f\right)=\psi(n) \Omega_{\chi}(f)$ for $f \in I(\chi)$ and $n \in N$.

2. For all $f \in I(\chi)$ with support on $P w_{\ell} P$, one has

$$
\Omega_{\chi}(f)=\int_{N} f\left(w_{\ell} n\right) \psi^{-1}(n) d n
$$

where the Haar measure on $N$ is normalized so that $\int_{N \cap K} d n=1$.

Let $\varphi_{K}$ denote the $K$-fixed element in $I(\chi)$ such that $\varphi_{K}(1)=1$. Then the unramified Whittaker function corresponding to $\chi, W_{\chi}: G \rightarrow \mathbb{C}$, is defined by

$$
W_{\chi}(g)=\Omega_{\chi}\left(R_{g} \varphi_{\chi}\right)
$$


and satisfies the condition

$$
W_{\chi}(n g k)=\psi(n) W_{\chi}(g) \quad \text { for } n \in N, g \in G \text { and } k \in K .
$$

For each root $\alpha \in \Sigma$, let $a_{\alpha}=\alpha^{\vee}(\varpi)$ where $\alpha^{\vee}$ is the coroot corresponding to $\alpha$. Then let

$$
\zeta(\chi)=\prod_{\alpha \in \Sigma^{+}}\left(1-q^{-1} \chi\left(a_{\alpha}\right)\right)
$$

By the Iwasawa decomposition $G=N A K$, the function $W_{\chi}$ is determined by its restriction to $A$. Let

$$
A^{-}=\{a \in A|| \alpha(a) \mid \leq 1 \text { for all } \alpha \in \Delta\} .
$$

Then we have $W_{\chi}(a)=0$ for $a \in A$ such that $a \notin A^{-}$. As for $a \in A^{-}$, we have the explicit formula [3]:

$$
W_{\chi}(a)=\zeta(\chi) \cdot \delta_{P}(a)^{\frac{1}{2}} \sum_{w \in W} w \chi(a) \cdot \prod_{\alpha \in \Sigma^{+}} \frac{1}{1-w \chi\left(a_{\alpha}\right)^{-1}}
$$

and $W_{\chi}(1)=\zeta(\chi)$. Let $W_{\chi}^{\circ}$ denote the normalized unramified Whittaker function defined by

$$
W_{\chi}^{\circ}=\zeta(\chi)^{-1} \cdot W_{\chi}
$$

3.2. Plancherel measure. Let $\mathcal{W}$ be the space of functions $h: G \rightarrow \mathbb{C}$ satisfying:

1. $h(n g k)=\psi(n)^{-1} \cdot h(g)$ for $n \in N, g \in G$ and $k \in K$.

2. $|h|$ is compactly supported on $N \backslash G$.

Let $\hat{A}$ denote the group of unramified characters of $A$. Then for $h \in \mathcal{W}$ we define its Fourier transform $\hat{h}: \hat{A} \rightarrow \mathbb{C}$ by

$$
\hat{h}(\chi)=\int_{N \backslash G} h(g) W_{\chi}^{\circ}(g) d g
$$

where the measure on $N \backslash G$ is normalized so that $\int_{N \backslash N K} d g=1$.

Let us define a measure $\nu$ on $\hat{A}$ by

$$
d \nu(\chi)=\frac{1}{|W|} \cdot \frac{d \chi}{|\epsilon(\chi)|^{2}}
$$

where $d \chi$ is the Haar measure on $\hat{A}$ normalized so that $\int_{\hat{A}} d \chi=1$ and

$$
\epsilon(\chi)=\prod_{\alpha \in \Sigma^{+}} \frac{1}{1-\chi\left(a_{\alpha}\right)^{-1}}
$$

Theorem 3. For $h \in \mathcal{W}$, we have

$$
h(g)=\int_{\hat{A}} \hat{h}(\chi) \overline{W_{\chi}^{\circ}(g)} d \nu .
$$

Proof. Let us call a double coset $N g K$ relevant when

$$
\psi(n)=1 \quad \text { for } n \in N \cap g K g^{-1} \text {. }
$$

We may take

$$
\Lambda^{-}=A^{-} / \mathbf{A}(\mathcal{O})
$$

as a set of representatives for relevant double cosets. Then each function $h \in \mathcal{W}$ is supported on a finite union of relevant double cosets and by an argument similar 
to the one in the proof of Theorem 1 it is enough to show that for $a, a^{\prime} \in \Lambda^{-}$, we have

$$
\frac{1}{|W|} \int_{\hat{A}} W_{\chi}^{\circ}(a) \overline{W_{\chi}^{\circ}\left(a^{\prime}\right)} \frac{d \chi}{|\epsilon(\chi)|^{2}}= \begin{cases}\delta_{P}(a), & \text { if } a=a^{\prime}, \\ 0, & \text { if } a \neq a^{\prime} .\end{cases}
$$

Since $|\epsilon(\chi)|^{2}=\epsilon(\chi) \epsilon\left(\chi^{-1}\right)$ is $W$-invariant, we have

$$
\begin{aligned}
\frac{1}{|\epsilon(\chi)|^{2}} W_{\chi}^{\circ}(a) \overline{W_{\chi}^{\circ}\left(a^{\prime}\right)}=\delta_{P}\left(a a^{\prime}\right)^{\frac{1}{2}} \cdot \sum_{w \in W} \frac{w \chi(a)}{\epsilon\left(w \chi^{-1}\right)} \cdot \sum_{\sigma \in W} \epsilon\left(\sigma \chi^{-1}\right) \sigma \chi\left(a^{\prime}\right)^{-1} \\
=\delta_{P}\left(a a^{\prime}\right)^{\frac{1}{2}} \sum_{w \in W} \sum_{\sigma \in W} w \chi(a) \cdot \sigma w \chi\left(a^{\prime}\right)^{-1} \cdot \prod_{\alpha \in \Sigma^{+}} \frac{1-w \chi\left(a_{\alpha}\right)}{1-\sigma w \chi\left(a_{\alpha}\right)} .
\end{aligned}
$$

Here we have

$$
\prod_{\alpha \in \Sigma^{+}} \frac{1-w \chi\left(a_{\alpha}\right)}{1-\sigma w \chi\left(a_{\alpha}\right)}=\operatorname{sgn}(\sigma) \prod_{\alpha \in \Sigma^{+}, \sigma \alpha<0} w \chi\left(a_{\alpha}\right)
$$

as shown in [3 page 227]. Since the Haar measure $d \chi$ is $W$-invariant, we have

$$
\begin{aligned}
& \frac{1}{|W|} \int_{\hat{A}} W_{\chi}^{\circ}(a) \overline{W_{\chi}^{\circ}\left(a^{\prime}\right)} \frac{d \chi}{|\epsilon(\chi)|^{2}} \\
& \quad=\delta_{P}\left(a a^{\prime}\right)^{\frac{1}{2}} \sum_{\sigma \in W} \operatorname{sgn}(\sigma) \int_{\hat{A}} \chi\left(a \prod_{\alpha \in \Sigma^{+}, \sigma \alpha<0} a_{\alpha}\right) \cdot \chi\left(\sigma^{-1} a^{\prime} \sigma\right)^{-1} d \chi
\end{aligned}
$$

Suppose that $\sigma \neq 1$ in (24). Let $\sigma=s_{n} s_{n-1} \cdots s_{1}$ be a minimal expression of $\sigma$ as a product of reflections corresponding to simple roots where $s_{i}$ is a reflection corresponding to $\alpha_{i} \in \Delta$ for $1 \leq i \leq n$. Then the positive roots made negative by $\sigma$ are

$$
\beta_{1}=\alpha_{1}, \quad \beta_{j}=s_{1} \cdots s_{j-1}\left(\alpha_{j}\right) \text { for } 2 \leq j \leq n .
$$

Let $\rho^{\vee}=\frac{1}{2} \sum_{\alpha>0} \alpha^{\vee}$. Then since

$$
\beta_{1}^{\vee}=\rho^{\vee}-s_{1} \rho^{\vee}, \quad \beta_{j}^{\vee}=s_{1} \cdots s_{j-1}\left(\rho^{\vee}-s_{j} \rho^{\vee}\right) \text { for } 2 \leq j \leq n,
$$

we have

$$
\sum_{j=1}^{n} \beta_{j}^{\vee}=\rho^{\vee}-s_{1} \cdots s_{n} \rho^{\vee}=\rho^{\vee}-\sigma^{-1} \rho^{\vee} .
$$

Let $\langle$,$\rangle denote the natural pairing between the group of rational characters$ $X^{*}(\mathbf{A})$ of $\mathbf{A}$ and the group of one-parameter subgroups $X_{*}(\mathbf{A})$ of $\mathbf{A}$. Then we note that $\left\langle\beta, \rho^{\vee}\right\rangle=1$ for all $\beta \in \Delta$ since

$$
s_{\beta}\left(\rho^{\vee}\right)=\rho^{\vee}-\left\langle\beta, \rho^{\vee}\right\rangle \beta^{\vee}=\rho^{\vee}-\beta^{\vee} .
$$

Hence we have

$$
\sum_{\alpha \in \Sigma^{+}, \sigma \alpha<0}\left\langle\alpha_{1}, \alpha^{\vee}\right\rangle=\sum_{j=1}^{n}\left\langle\alpha_{1}, \beta_{j}^{\vee}\right\rangle=\left\langle\alpha_{1}, \rho^{\vee}-\sigma^{-1} \rho^{\vee}\right\rangle=1-\left\langle\sigma \alpha_{1}, \rho^{\vee}\right\rangle \geq 2
$$

since $\sigma \alpha_{1}<0$. Let $\gamma=-\sigma \alpha_{1}$. Then $\gamma$ is a positive root and

$$
\sigma^{-1} \gamma\left(a \prod_{\alpha \in \Sigma^{+}, \sigma \alpha<0} a_{\alpha}\right)=\alpha_{1}(a)^{-1} \varpi^{-\sum_{\alpha \in \Sigma^{+}, \sigma \alpha<0}\left\langle\alpha_{1}, \alpha^{\vee}\right\rangle}
$$


Hence

$$
a \prod_{\alpha \in \Sigma^{+}, \sigma \alpha<0} a_{\alpha} \notin \sigma^{-1} A^{-} \sigma .
$$

Thus only $\sigma=1$ contributes to (24) and we have (23).

3.3. Inversion formula for the Gelfand transform. Let $\mathcal{H}(=\mathcal{H}(G, K))$ denote the Hecke algebra of $G$ with respect to $K$. Then for $f \in \mathcal{H}$, we define its Gelfand transform $\Psi_{f}: G \rightarrow \mathbb{C}$ by

$$
\Psi_{f}(g)=\int_{N} f(g n) \psi^{-1}(n) d n
$$

Theorem 4. For $a \in \Lambda^{-}$, we have

$$
\Psi_{f}\left(a^{-1}\right)=\delta_{P}(a)^{\frac{1}{2}} \sum_{\alpha \in \Sigma^{+}} \sum_{m_{\alpha} \in\{0,1\}}(-1)^{\sum_{\alpha \in \Sigma^{+}} m_{\alpha}} S f\left(a^{-1} \prod_{\alpha \in \Sigma^{+}} a_{\alpha}^{-m_{\alpha}}\right)
$$

where $S f$ denotes the Satake transform of $f$ defined by

$$
S f(b)=\delta_{P}(b)^{\frac{1}{2}} \int_{A} f(b n) d n, \quad b \in A .
$$

Proof. By an argument similar to the one in the proof of Theorem 2 we have

$$
\Psi_{f}\left(a^{-1}\right)=\sum_{b \in A / \mathbf{A}(\mathcal{O})} S f(b) K(a, b)
$$

where

$$
K(a, b)=\int_{\hat{A}} \chi^{-1}(b) \overline{W_{\chi}^{\circ}(a)} d \nu=\frac{\delta_{P}(a)^{\frac{1}{2}}}{|W|} \sum_{w \in W} K_{w}(a, b)
$$

and

$$
K_{w}(a, b)=\int_{\hat{A}} w \chi\left(a^{-1} \cdot w b^{-1} w^{-1}\right) \prod_{\alpha \in \Sigma^{+}}\left(1-w \chi\left(a_{\alpha}\right)^{-1}\right) d \chi
$$

By the Schur orthogonality, we have

$$
K_{w}(a, b)=\sum_{\alpha \in \Sigma^{+}} \sum_{m_{\alpha} \in\{0,1\}}(-1)^{\sum_{\alpha \in \Sigma^{+}} m_{\alpha}} \delta\left(a^{-1} \prod_{\alpha \in \Sigma^{+}} a_{\alpha}^{-m_{\alpha}}, w b w^{-1}\right)
$$

where

$$
\delta\left(a_{1}, a_{2}\right)= \begin{cases}1, & \text { when } a_{1} \equiv a_{2} \quad(\bmod \mathbf{A}(\mathcal{O})) \\ 0, & \text { otherwise }\end{cases}
$$

for $a_{1}, a_{2} \in A$. Since $S f\left(w b w^{-1}\right)=S f(b)$ for $b \in A$ and $w \in W$, we have (25).

The formula (25) has been proved by Ye [8] for GL $(n)$ using a different method. 


\section{REFERENCES}

1. D. Bump, S. Friedberg and M. Furusawa, Explicit formulas for the Waldspurger and Bessel models, Israel J. Math. 102 (1997), 125-177. MR 98k:22070

2. W. Casselman, The unramified principal series of $\mathfrak{p}$-adic groups I. The spherical function, Compositio Math. 40 (1980), 387-406. MR 83a:22018

3. W. Casselman and J. Shalika, The unramified principal series of p-adic groups II. The Whittaker function, Compositio Math. 41 (1980), 207-231. MR 83i:22027

4. M. Furusawa and J. A. Shalika, On central critical values for the degree four L-functions for GSp (4): The fundamental lemma, preprint.

5. I. G. Macdonald, Spherical functions on a group of p-adic type, Publications of the Ramanujan Institute, No. 2. Ramanujan Institute, Center for Advanced Study in Mathematics, University of Madras, Madras, 1971. MR 55:8261

6. M. Novodvorsky, New unique models of representations of unitary groups, Compositio Math. 33 (1976), 289-295. MR 58:11244

7. Y. Ye, The fundamental lemma of a relative trace formula for GL (3), Compositio Math. 89 (1993), 121-162. MR 95b:22023

8. Y. Ye, An integral transform and its applications, Math. Ann. 300 (1994), 405-417. MR 95j:11045

Department of Mathematics, Graduate School of Science, Osaka City University, Sugimoto 3-3-138, SumiYoshi-Ku, OSAKa 558-8585, JaPAN

E-mail address: furusawa@sci.osaka-cu.ac.jp

Department of Mathematics, The Johns Hopkins University, 3400 North Charles Street, Baltimore, Maryland 21218

E-mail address: shalika@math.jhu.edu 